\title{
Origin of the transition entropy in vanadium dioxide
}

\author{
Thomas A. Mellan, ${ }^{1, *}$ Hao Wang, ${ }^{2}$ Udo Schwingenschlögl, ${ }^{2}$ and Ricardo Grau-Crespo ${ }^{3}$ \\ ${ }^{1}$ Thomas Young Centre for Theory and Simulation of Materials, Department of Materials, Imperial College London, \\ Exhibition Road, London SW7 2AZ, United Kingdom \\ ${ }^{2}$ King Abdullah University of Science and Technology (KAUST), Physical Science and Engineering Divison (PSE), \\ Thuwal 23955-6900, Saudi Arabia \\ ${ }^{3}$ Department of Chemistry, University of Reading, Whiteknights, Reading RG6 6AD, United Kingdom
}

(Received 27 September 2018; revised manuscript received 14 January 2019; published 26 February 2019)

\begin{abstract}
The reversible metal-insulator transition in $\mathrm{VO}_{2}$ at $T_{\mathrm{c}} \approx 340 \mathrm{~K}$ has been closely scrutinized yet its thermodynamic origin remains ambiguous. We discuss the origin of the transition entropy by calculating the electron and phonon contributions at $T_{\mathrm{c}}$ using density functional theory. The vibration frequencies are obtained from harmonic phonon calculations, with the soft modes that are imaginary at zero temperature renormalized to real values at $T_{\mathrm{c}}$ using experimental information from diffuse $\mathrm{x}$-ray scattering at high-symmetry wave vectors. Gaussian process regression is used to infer the transformed frequencies for wave vectors across the whole Brillouin zone, and in turn compute the finite temperature phonon partition function to predict transition thermodynamics. Using this method, we predict the phase transition in $\mathrm{VO}_{2}$ is driven 5 to 1 by phonon entropy over electronic entropy, and predict a total transition entropy that agrees (within 5\%) with the calorimetric value.
\end{abstract}

DOI: 10.1103/PhysRevB.99.064113

\section{INTRODUCTION}

The first-order phase transition in $\mathrm{VO}_{2}$ occurs at a temperature of $T_{\mathrm{c}} \approx 340 \mathrm{~K}$, and is coupled to defect concentration [1-6], strain field [7-9], electric field [10], and optical fluence $[11,12]$. The transition has been studied since Klemm and Grimm in the 1930s [13], Cook in the 1940s [14], and in detail by Morin in 1959 [15]. Fundamental questions on the nature of the transition have been debated for decades [16-19], and continue to be researched [20-22]. The transition occurs most notedly in temperature so understanding the thermodynamic origin is a point of basic importance.

In this study we use density functional theory (DFT) to predict the origin of the $\mathrm{VO}_{2}$ transition entropy. The applicability of DFT to describe the transition metal oxide class of solids depends sensitively on technical details $[23,24]$. We use non-spin-polarized calculations based on the PBE exchange correlation functional [25], with on-site Coulomb correction $U_{\text {eff }}=3 \mathrm{eV}$ [26]. As shown in Fig. 1, this approach leads to agreement with experiment on the following important points:

(1) Electronic structure-the high-symmetry metallic $R$ phase is appropriately gapless. Band gap is opened smoothly with $\mathrm{V}-\mathrm{V}$ dimerization, resulting in a semiconducting monoclinic $(M 1)$ phase.

(2) Transition enthalpy-the low-temperature $M 1$ phase is energetically favored over the high-temperature $R$ phase [27].

(3) Mechanical stability - the low-symmetry $M 1$ phase is stable against distortion and the high-symmetry $R$ phase is unstable in 0-K DFT simulation.

Including spin polarization is shown in Fig. 1 to lower the DFT energy of $R-\mathrm{VO}_{2}$ with respect to the nonmagnetic solution, destroying agreement with experiment for the points

*t.mellan@imperial.ac.uk listed above. The problems related with spin polarization in the DFT description of $\mathrm{VO}_{2}$ have been discussed before [28], and have been resolved fully only in the context of quantum Monte Carlo simulations [20], which are too computationally expensive to use to investigate lattice dynamics. We therefore take the pragmatic approach employed by other authors of using nonmagnetic calculations [29], on the basis of their agreement with experiment.

For the $M 1$ phase, the Born-Oppenheimer surface is convex about equilibrium coordinates. The harmonic approximation to the interatomic potential is appropriate for small displacements, and is expected to be adequate for $M 1-\mathrm{VO}_{2}$ up to $T_{\mathrm{c}}$. On the other hand the high-symmetry $R$ phase has negative second-order force constants which qualitatively invalidate free-energy predictions at the harmonic level. Approaches to remedy this that include anharmonic effects have become more accessible thanks to recent developments [30-35], enabling the description of systems with light atoms, at ultrahigh temperatures, or near phase transitions, yet widespread application of first-principles anharmonic thermodynamics remains limited due to computational cost and complexity. In this work we present a simple, experimentally motivated approach to compute the thermodynamics of temperaturestabilized imaginary modes in $\mathrm{VO}_{2}$. The method is low cost and applicable generally to the DFT thermodynamics of hightemperature phases that are unstable at zero temperature.

\section{SOFT MODE THEORETICAL APPROACH}

In the soft mode theory of Cochran and in Landau phenomenological approaches [37-40], a square-root temperature dependence is identified for transition parameters. The squared frequency $\tilde{\omega}_{i \mathbf{q}}^{2}$ of a mode $i$ that softens near the transition at wave vector $\mathbf{q}$ is expected to decrease 


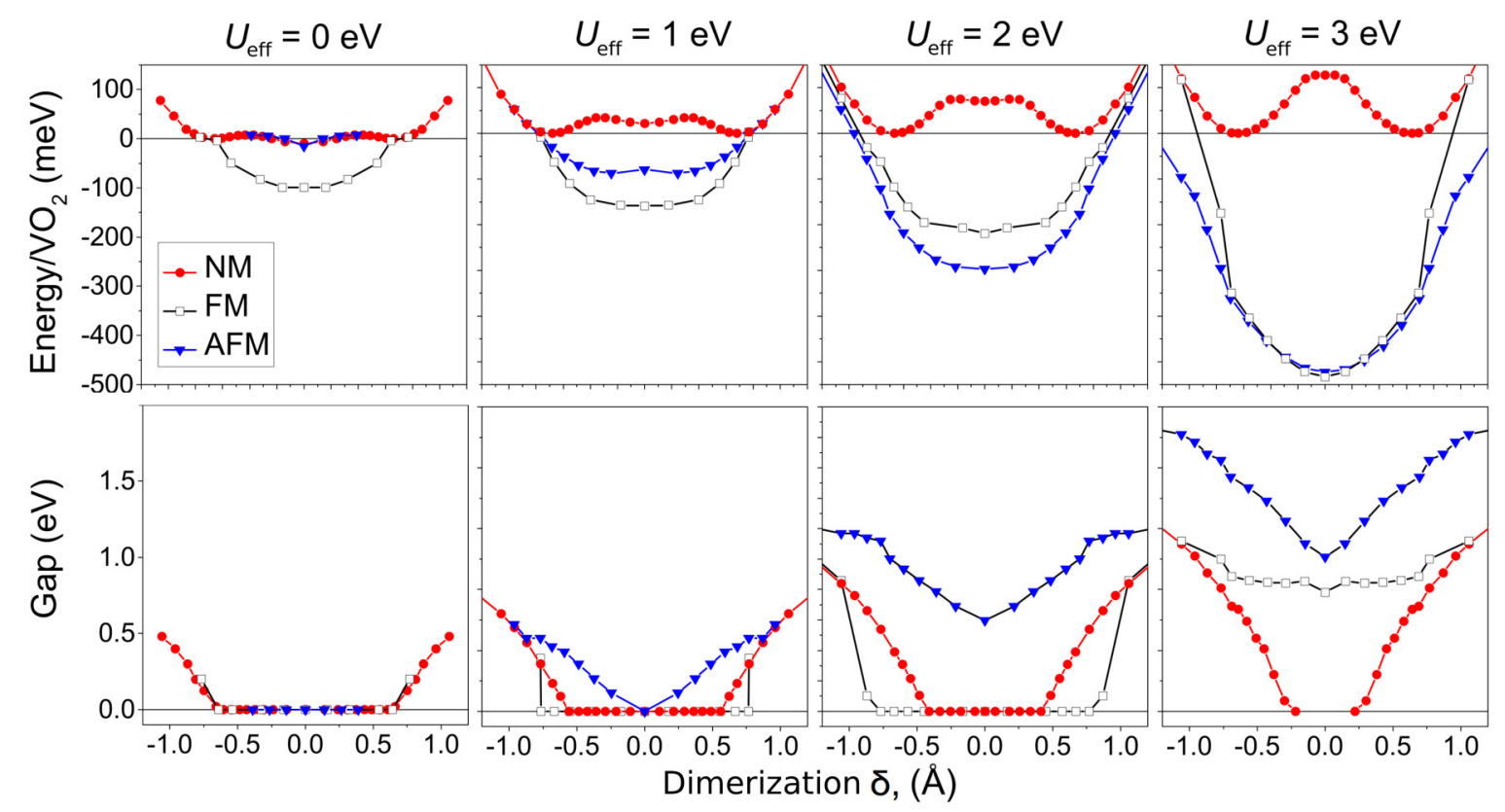

FIG. 1. Potential-energy surface $\left(\mathrm{eV} / \mathrm{VO}_{2}\right)$ and band gap $(\mathrm{eV})$ as a function of dimerization $\delta(\AA)$, which is the difference in $d(\mathrm{~V}-\mathrm{V})$ between consecutive pairs of cations along the rutile $c$ axis. Values are shown for nonmagnetic (NM), antiferromagnetic (AFM), and ferromagnetic (FM) ordering, and for a range of $d$ electron on-site Coulomb interaction strengths, $U_{\text {eff }}=[0,3] \mathrm{eV}$. The NM $U_{\text {eff }}=3 \mathrm{eV}$ description appropriately opens the band gap in dimerization and provides a mechanically unstable high-symmetry phase.

linearly with temperature towards a first-order solid-state transition [41]:

$$
\tilde{\omega}_{i \mathbf{q}}^{2}(T) \propto T-T_{0} .
$$

In $\mathrm{VO}_{2}$ this proportionality has been observed in experimental measurements [36]. For example, Cohen and Terauchi report a linear temperature response from diffuse $\mathrm{x}$-ray-scattering measurements at $\mathbf{q}=\mathbf{R}$ with $\mathbf{R}=\left\langle\frac{1}{2} 0 \frac{1}{2}\right\rangle$ which is represented in the left axis in Fig. 2. The temperature $T_{0}$, when $\tilde{\omega}_{i \mathbf{R}} \rightarrow 0$, has a value of $T_{0}=329 \mathrm{~K}$, and corresponds to the classical second-order transition temperature. Along with the firstorder transition temperature $T_{\mathrm{c}}$, Eq. (1) relates the phonon frequency at $0 \mathrm{~K}, \tilde{\omega}_{i \mathrm{R}}^{2}(0)$, to the frequency at the transition temperature as

$$
\tilde{\omega}_{i \mathrm{R}}^{2}\left(T_{\mathrm{c}}\right)=\tilde{\omega}_{i \mathrm{R}}^{2}(0) \frac{T_{\mathrm{c}}-T_{0}}{-T_{0}} .
$$

Here the shifted frequency $\tilde{\omega}_{i \mathrm{R}}(0)$ is equal to the harmonic frequency $\omega_{i \mathbf{R}}$ that is calculated with DFT. The application of the transformation of imaginary harmonic DFT frequencies at $0 \mathrm{~K}$ to real frequencies at $T_{\mathrm{c}}$ is shown in Fig. 2. The method gives values for the temperature-stabilized frequencies at negligible additional cost to standard harmonic DFT calculations, provided the coefficients $T_{0}$ and $T_{\mathrm{c}}$ are known, which is often the case as shown by the experimental data reviewed by Cochran and Cowley $[40,41]$.

To implement the frequency shifts for $R-\mathrm{VO}_{2}$, the transition modes which are shown in Fig. 2 to be imaginary at $\mathbf{R}, \mathbf{Z}$, and $\mathbf{A}$ in $q_{z}=\frac{1}{2}$ are renormalized to $T_{\mathrm{c}}$ following the prescription described in the previous paragraph. At other $R-\mathrm{VO}_{2}$ wave vectors (see Brillouin-zone geometry, Fig. 3, Appendix B), the frequencies do not soften to imaginary harmonic frequencies. For example, in Fig. 2 the $\boldsymbol{\Gamma}, \mathbf{X}$, and $\mathbf{M}$ wave vectors in $q_{z}=0$ have real harmonic frequencies at $T=0 \mathrm{~K}$. The frequencies in $q_{z}=0$ that do not soften are modelled using the DFT harmonic frequencies $\omega_{i \mathbf{q}}$.

In order to make thermodynamic predictions for a hightemperature phase we need to sample the transformed modes finely across the Brillouin zone, not only at the limited high-symmetry $\mathbf{q}$ points in the $q_{z}=\frac{1}{2}$ and $q_{z}=0$ regions described. To obtain $\tilde{\omega}_{i \mathbf{q}}\left(T_{\mathrm{c}}\right) \in \mathbb{R} \forall i \mathbf{q}$, the partial knowledge we already have of the frequencies transformed to $T_{\mathrm{c}}$ is found to be sufficient data for machine learning techniques to interpolate $\tilde{\omega}_{i \mathbf{q}}\left(T_{\mathrm{c}}\right)$ to arbitrary phonon wave vectors. $\tilde{\omega}_{i \mathbf{q}}\left(T_{\mathrm{c}}\right)$ is inferred at all irreducible Brillouin-zone wave vectors using Gaussian process regression (GPR) [42], enabling the partition function of the $R-\mathrm{VO}_{2}$ vibrational system to be specified at $T_{\mathrm{c}}$. GPR accuracy benchmarks and technical details are provided in Appendix D.

To understand the source of entropy driving the transition, we compute $S_{\mathrm{DFT}}=S_{\mathrm{R}}-S_{\mathrm{M} 1}$ at $T_{\mathrm{c}}$. $S_{\mathrm{M} 1}$ is the DFT harmonic vibrational entropy of $M 1-\mathrm{VO}_{2}$, and for $R-\mathrm{VO}_{2}$ the entropy is calculated as $S_{\mathrm{R}}=S_{\mathrm{R}}^{\mathrm{el}}+S_{\mathrm{R}}^{\mathrm{ph}}+\tilde{S}_{\mathrm{R}}^{\mathrm{ph}}$ with consecutive terms from electrons, the harmonic phonon entropy, and the softmode phonon entropy from the two experimentally renormalized transition modes. Thermodynamic calculation details are provided in Appendix C.

\section{RESULTS AND DISCUSSION}

The total transition entropy we predict for $\mathrm{VO}_{2}$ is $S_{\mathrm{DFT}}=$ $1.42 k_{\mathrm{B}} / \mathrm{VO}_{2}$. The commonly referenced calorimetric value is $S_{\text {exp }}=1.5 k_{\mathrm{B}} / \mathrm{VO}_{2}$ [43]. Our predicted value of $S_{\mathrm{DFT}}=$ $1.42 k_{\mathrm{B}} / \mathrm{VO}_{2}$ agrees with the calorimetric value to within $5 \%$. The predicted value is composed of the contributions $S^{\mathrm{el}}=$ 0.25 and $S^{\mathrm{ph}}=1.17 k_{\mathrm{B}} / \mathrm{VO}_{2}$. The source of entropy driving the transition is therefore phonons over electrons at a ratio of almost 5 to 1 . 


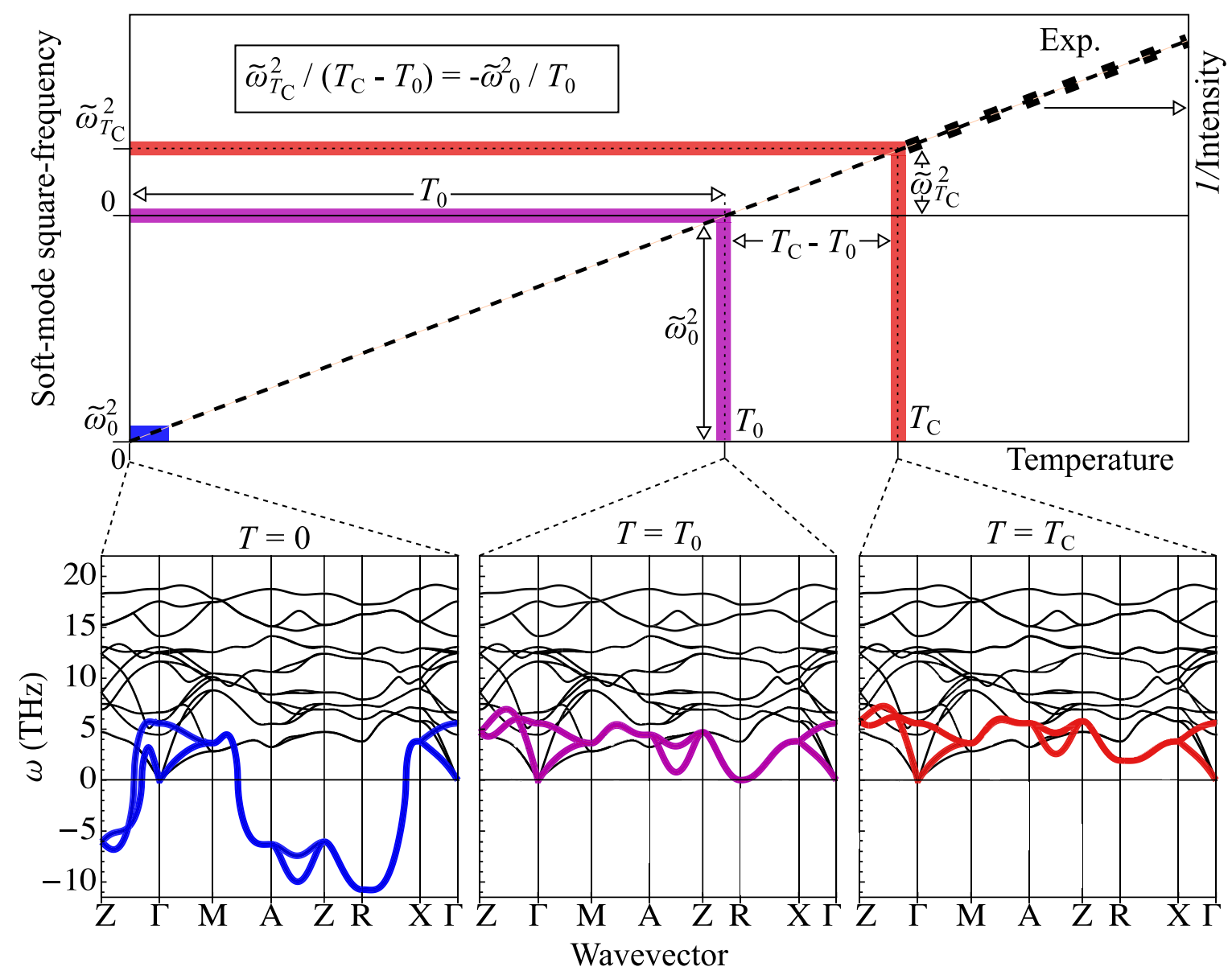

FIG. 2. Top, right axis: Inverse intensity from diffuse x-ray scattering vs temperature [36]. Top, left axis: Linear softening of square frequencies with temperature. The transition occurs at $T_{\mathrm{c}}$ and the classical second-order transition temperature is $T_{0}$. The frequencies at $0 \mathrm{~K}$ and $T_{\mathrm{c}}$, shown as $\tilde{\omega}_{0}$ and $\tilde{\omega}_{T_{\mathrm{c}}}$, are related linearly. Bottom: DFT phonon dispersion for $R-\mathrm{VO}_{2}$ at $0 \mathrm{~K}, T_{0}$ and $T_{\mathrm{c}}$. Thick colored lines show the soft modes after renormalization, at $0 \mathrm{~K}$ (blue), $T_{0}$ (magenta), and $T_{\mathrm{c}}$ (red).

In Table I our entropy predictions are compared to 14 recent and historically reported values from the literature. The values range widely, from $S^{\mathrm{el}}=0.01$ to $0.6 k_{\mathrm{B}} / \mathrm{VO}_{2}$ and $S^{\mathrm{ph}}=0.64$ to $1.35 k_{\mathrm{B}} / \mathrm{VO}_{2}$. Among the range of predictions, our conclusions align most closely with those of Budai et al. [44], with which we agree that the transition thermodynamics are mostly due to phonons. One difference is that Budai et al. [44] compute a phonon entropy of $0.93 k_{\mathrm{B}} / \mathrm{VO}_{2}$ compared to $1.17 k_{\mathrm{B}} / \mathrm{VO}_{2}$ here. Their value is based on $U_{\text {eff }}=0$ $\mathrm{eV}$ calculations, whereas this work uses $U_{\text {eff }}=3 \mathrm{eV}$ DFT, to ensure a qualitatively correct description of the electron band gap, transition enthalpy, and $R$-point lattice instability. (Note that the sensitivity of $S^{\mathrm{ph}}$ is less than $1 \%$ per $0.1 \mathrm{eV}$ of $U_{\text {eff }}$ about the appropriate value of $U_{\text {eff }}=3 \mathrm{eV}$, but the $U_{\text {eff }}=0 \mathrm{eV}$ description has qualitatively incorrect features.) A second difference with the results of Budai et al. [44] concerns their reported "best" predictions, which use a phonon entropy estimate from scattering measurements, which is $1.02 \pm 0.09 k_{\mathrm{B}} / \mathrm{VO}_{2}$, compared to our value of $1.17 k_{\mathrm{B}} / \mathrm{VO}_{2}$. Consequently the error with respect to the total calorimetric value [43] of $1.5 \pm 0.01 k_{\mathrm{B}} / \mathrm{VO}_{2}$ is $0.08 k_{\mathrm{B}} / \mathrm{VO}_{2}$ here, rather than $0.21 k_{\mathrm{B}} / \mathrm{VO}_{2}$ [44].

A controversial point that merits discussion is the possibility of a spin contribution to the transition entropy. Quantum
Monte Carlo calculations have predicted that the $R$ phase, which in nature only exists above $T_{\mathrm{c}}$, would be spin ordered at $T=0 \mathrm{~K}$ [20]. On this basis Xia and Chen suggest a spin contribution to the transition entropy [45]. Accounting for a coincident spin disordering at $T_{\mathrm{c}}$ in our predictions increases the entropy value by $\ln (2)=0.69 k_{\mathrm{B}} / \mathrm{VO}_{2}$ to $S_{\mathrm{DFT}}=S^{\text {el }}+$ $S^{\mathrm{ph}}+S^{\text {spin }}=0.25+1.17+0.69=2.11 k_{\mathrm{B}} / \mathrm{VO}_{2}$, which exceeds the experimental value of $S_{\exp }=1.5 k_{\mathrm{B}} / \mathrm{VO}_{2}$. If a $\ln (2)$ spin contribution to the transition entropy exists, $S^{\text {ph }}$ must be considerably lower for $S_{\mathrm{DFT}}$ to remain consistent with $S_{\text {exp }}$. Considering the neutron-scattering measurements by Budai et al. we are inclined to believe this is unlikely [44]. The neutron measured phonon density of states (DOS) can be used to estimate a phonon entropy of $1.02 k_{\mathrm{B}} / \mathrm{VO}_{2}$ (Table I), which is similar to our predicted value of $S^{\mathrm{ph}}=1.17 \mathrm{k}_{\mathrm{B}} / \mathrm{VO}_{2}$, and insufficiently small to accommodate the full spin term. We therefore consider that a fully disordered Heisenberg spin contribution to the transition is unlikely. In order to confirm or refute inferences based on our experimentally renormalized DFT thermodynamics and the neutron-scattering measurements of Budai et al. [44], we propose a simple experiment to measure $T_{\mathrm{c}}$ in the presence of a strong magnetic field. If there is a spin contribution to the entropy, it should vanish in the presence of the magnetic field, which will bring the value 
TABLE I. Historical measured and computed $\mathrm{VO}_{2}$ transition entropies, $S\left(k_{\mathrm{B}} / \mathrm{VO}_{2}\right)$, along with available partial electron, phonon, and spin contributions. * Unpublished measurements by Ryder, reported by Berglund et al. [43]. § Values determined from analysis of Ryder's measurements [43]. ${ }^{\dagger}$ Mott and Zylbersztejn base their analysis on a total transition entropy of $S=1.6 k_{\mathrm{B}} / \mathrm{VO}_{2}$, mis-citing a Berglund report which has the entropy at $S=1020 \pm 5 \mathrm{cal} / \mathrm{mol}$ or $S=1.51 \pm 0.01 k_{\mathrm{B}} / \mathrm{VO}_{2}$, assuming $T=340.5 \pm 0.5 \mathrm{~K}$.

\begin{tabular}{|c|c|c|c|c|c|}
\hline \multirow[t]{2}{*}{ Source } & \multirow[t]{2}{*}{ Method } & \multicolumn{4}{|c|}{ Entropy contributions } \\
\hline & & $S^{\mathrm{ph}}$ & $S^{\mathrm{el}}$ & $S^{\text {spin }}$ & $S$ \\
\hline Klemm and Grimm [13], 1939 & calorimetry measurements & & & & 1.2 \\
\hline Kawakubo [46], 1964 & calorimetry measurements & & & & 1.1 \\
\hline Ryder [43], 1969 & calorimetry measurements & & & & $1.51 \pm 0.01^{*}$ \\
\hline Berglund et al. [43], 1969 & analysis of Ryder's heat capacity measurements & $1.25 \S$ & $0.25 \S$ & & $1.51 \pm 0.01^{*}$ \\
\hline Hearn [48], 1972 & 1D model calc. & 1.17 & 0.01 & & 1.18 \\
\hline Chandrasekhar et al. [49], 1973 & scanning calorimetry measurements & & & & 1.65 \\
\hline Zylbersztejn and Mott [16], 1975 & analysis of magnetic susceptibility measurements & 1.02 & 0.58 & & $1.6(1.51)^{\dagger}$ \\
\hline Pintchovski et al. [50], 1978 & calorimetry and electrical resistivity measurements & 0.9 & 0.6 & & - \\
\hline Maurer et al. [51], 1999 & Debye model fitted to sound velocity measurement & 1.35 & & & - \\
\hline Budai et al. [44], 2014 & IXS phonon measurements and DFT electron calc. & $1.02 \pm 0.09$ & 0.27 & & $1.29 \pm 0.09$ \\
\hline
\end{tabular}

of $T_{\mathrm{c}}$ significantly up. If there is no magnetic entropy involved in the transition, $T_{\mathrm{c}}$ should not change or change very little in the presence of the field.

\section{CONCLUSION}

We have described the source of entropy driving the $\mathrm{VO}_{2}$ metal-insulator transition. Our thermodynamic predictions suggest the transition is driven by phonons over electrons at a ratio of $1.17: 0.25$, and that the computed entropy agrees with the calorimetric entropy value to within $5 \%$. In order to make our predictions we have performed DFT harmonic phonon calculations, in conjunction with an experimentally motivated soft-mode renormalization scheme based on data from x-ray-scattering measurements. The scheme has predicted values of soft-mode frequencies at the transition temperature for high-symmetry points in the Brillouin zone of $R-\mathrm{VO}_{2}$. The machine learning interpolation method Gaussian process regression was used to infer the soft-mode frequencies across the full Brillouin zone based on the input of frequencies at partial high-symmetry wave vectors. A simple procedure has been proposed to experimentally confirm or refute claims of a spin disorder contribution to the transition entropy.

\section{ACKNOWLEDGMENTS}

R.G.-C. and T.A.M. acknowledge funding from the UK's Engineering and Physical Sciences Research Council EPSRC (Grant No. EP/J001775/1). Via the UK's HPC Materials Chemistry Consortium, which is funded by EPSRC (Grant No. EP/L000202), this work made use of ARCHER, the UK's national high-performance computing services. The research reported in this publication was supported by funding from King Abdullah University of Science and Technology (KAUST). T.A.M. is grateful for computational support from the UK Materials and Molecular Modelling Hub, which is partially funded by EPSRC (Grant No. EP/P020194), for which access was obtained via the UKCP consortium and funded by EPSRC Grant No. EP/P022561/1.

\section{APPENDIX A: DFT CALCULATIONS}

Periodic DFT calculations were performed with the Vienna $A b$-initio Simulation Package (VASP) [52,53], using the generalized gradient approximation (GGA) in the form of the Perdew-Burke-Ernzerhof exchange-correlation functional (PBE) [25]. The projected augmented wave method was used to describe the interaction between the valence electrons and the core states, which were kept frozen at the atomic references (up to $3 p$ in $\mathrm{V}$ and $1 s$ in $\mathrm{O}$ ) [54,55]. Plane waves were cut off at a kinetic energy of $520 \mathrm{eV}$, and $\mathbf{k}$ points were sampled at a density of $6 \times 6 \times 9$ divisions per rutile unit cell. Force and energy convergence thresholds were set to $10^{-3} \mathrm{eV} / \AA$ and $10^{-6} \mathrm{eV}$ respectively.

The Coulomb interaction between vanadium $d$ electrons was corrected with an effective on-site term, $U_{\text {eff }}$ [26]. The effect of $U_{\text {eff }}$ and magnetic ordering was considered for phase enthalpy and band gap. NM $U_{\text {eff }}=3 \mathrm{eV}$ calculations reproduce the basic characteristics well known from experiment, including instability of the high-symmetry $P 4_{2} / \mathrm{mnm}$ rutile phase at low temperature, and electronic band-gap phase opening with $\mathrm{V}-\mathrm{V}$ dimerization.

\section{APPENDIX B: PHONON CALCULATIONS}

Phonons were computed from second-order force constants using the PHONOPY code [56]. The $M 1$ and $R$ phases employ $2 \times 2 \times 2$ and $2 \times 2 \times 3$ supercells respectively. Phonon thermodynamic functions were satisfactorily converged at a sampling density equivalent to $16 \times 16 \times 24 \mathbf{q}-$ point mesh for the rutile conventional unit cell.

Harmonic DFT phonon dispersion is shown in Fig. 3. The Brillouin zones for $M 1$ and $R$ unit 

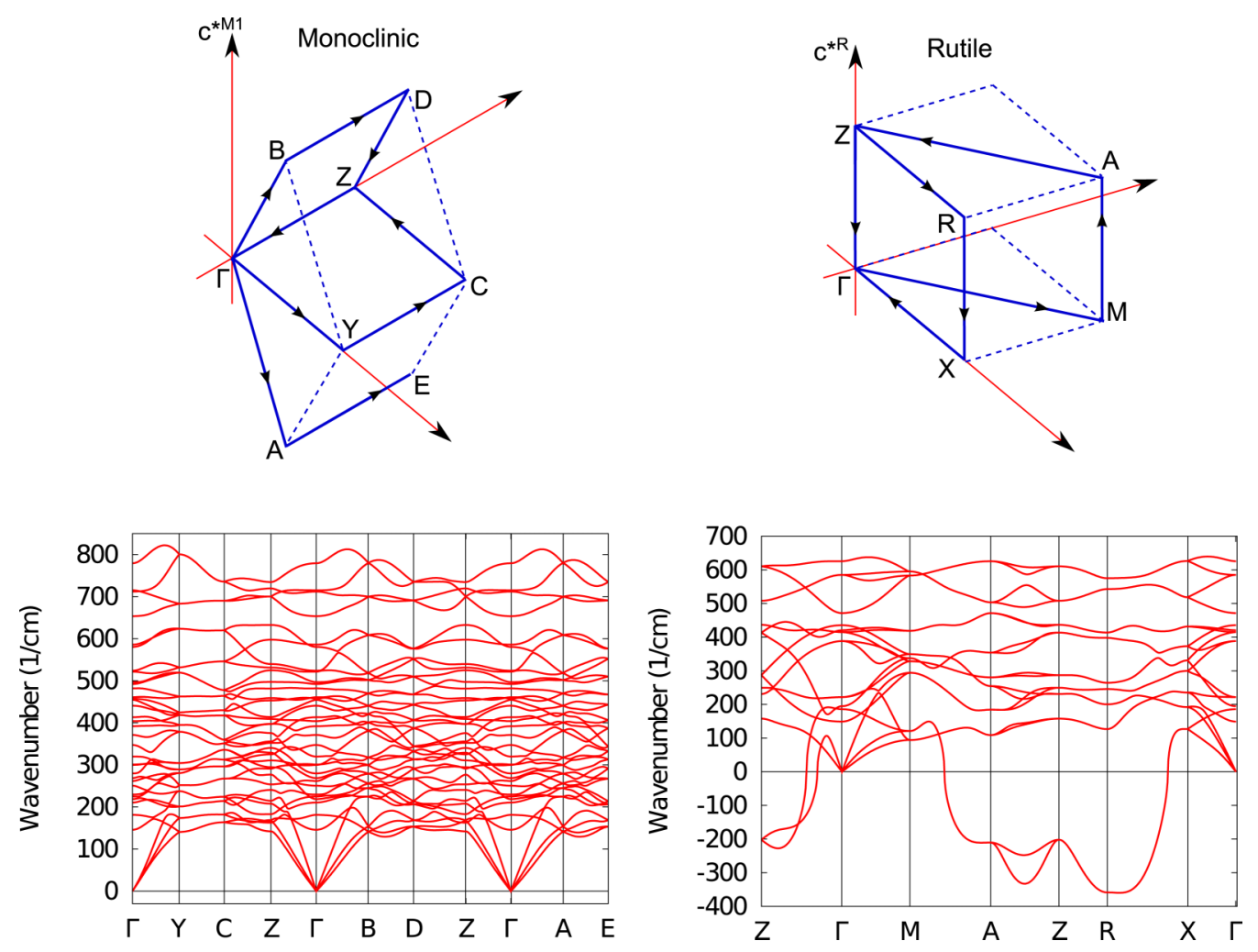

FIG. 3. DFT harmonic phonon dispersion for $M 1$ and $R-\mathrm{VO}_{2}$, and Brillouin-zone sampling paths.

cells are sampled between high-symmetry points in reciprocal space. The path for $R-\mathrm{VO}_{2}$ follows the sequence $\{Z, \Gamma, M, A, Z, R, X, \Gamma\}$ which corresponds to $\left\{00 \frac{1}{2}, 000, \frac{1}{2} \frac{1}{2} 0, \frac{1}{2} \frac{1}{2} \frac{1}{2}, 00 \frac{1}{2}, \frac{1}{2} 0 \frac{1}{2}, \frac{1}{2} 00,000\right\}$. The path for $M 1-\mathrm{VO}_{2}$ is $\{\Gamma, Y, C, Z, \Gamma, B, D, Z, \Gamma, A, E\}$ which corresponds to $\left\{000,0 \frac{1}{2} 0, \frac{1}{2} \frac{1}{2} 0, \frac{1}{2} 00,000,0 \frac{1}{4} \frac{1}{2}, \frac{1}{2} \frac{1}{4} \frac{1}{2}, \frac{1}{2} 00,000\right.$, $\left.0 \frac{1}{4} \frac{1}{2}, \frac{1}{2} \frac{1}{4} \frac{1}{2}\right\}$. The phonon densities of states for the $M 1$ and $R$ phases are shown projected by atomic species in Fig. 4. The eigenvectors of the imaginary transition modes are shown to project primarily onto the motion of vanadium atoms.

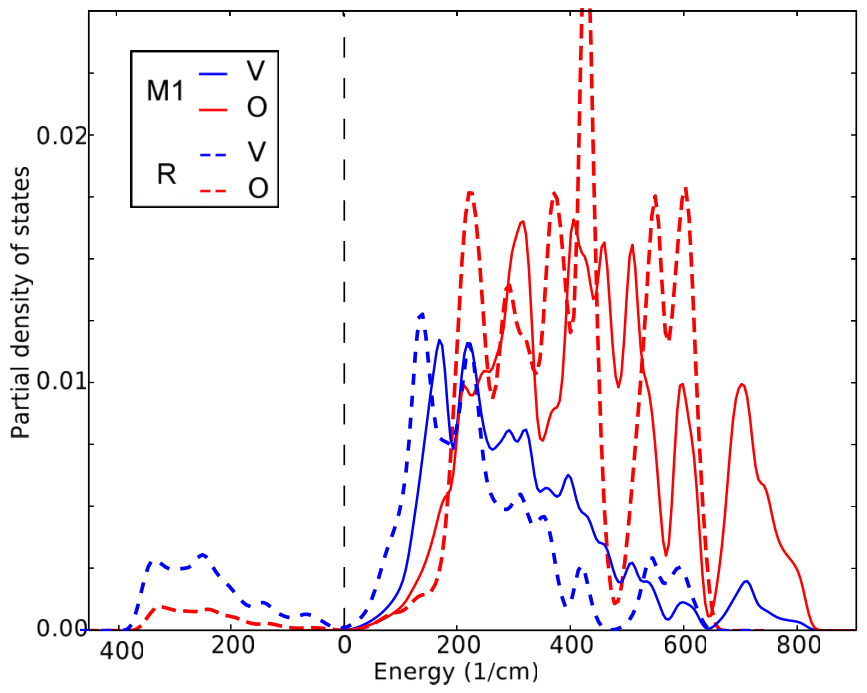

FIG. 4. DFT harmonic phonon density of states for $M 1$ and $R-\mathrm{VO}_{2}$.

\section{APPENDIX C: THERMODYNAMICS}

The phonon entropy difference between the $M 1$ and the $R$ phases is estimated from the harmonic free energy

$$
S^{\mathrm{ph}}=-\partial_{T} F^{\mathrm{ph}},
$$

where $F^{\mathrm{ph}}$ is

$$
F^{\mathrm{ph}}=-T \ln Z,
$$

and partition function is computed using the harmonic geometric series expression

$$
Z=\prod_{i \mathbf{q}} \frac{e^{-\beta \omega_{i \mathbf{q}} / 2}}{1-e^{-\beta \omega_{i \mathbf{q}}}},
$$

with $\beta \equiv T^{-1}$.

For the $M 1$ phase, entropy is calculated from the standard DFT harmonic frequencies, $\omega_{i q}$. For the $R$ phase the same expression is applied to the $3 n-2$ real harmonic DFT frequencies that don't soften at the transition, and the two shifted frequencies $\tilde{\omega}_{i \mathbf{q}}$, for the two imaginary harmonic modes subject to the experimental renormalization to real effective frequencies.

The $R$ phase is metallic. As we are only interested in thermal electron excitations at moderate temperatures we assume $\partial_{T} g(E)=0$, and that electronic entropy of the $R$ phase can be given in terms of partial one-electron occupancies as

$$
S^{\mathrm{el}}=-\int d E g(E)\{f \ln f+(1-f) \ln (1-f)\} .
$$



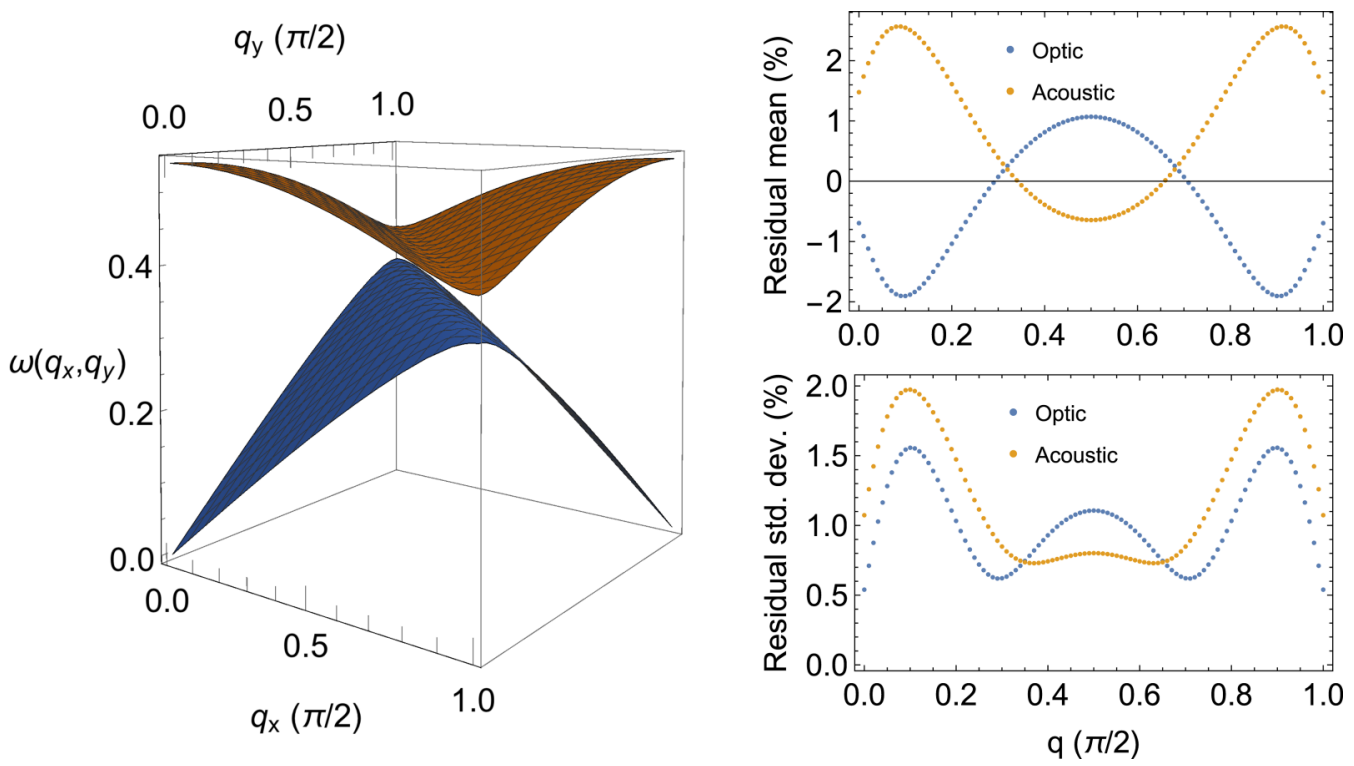

FIG. 5. Right: Analytic dispersion system used to benchmark Gaussian process regression (GPR) performance for Brillouin-zone interpolation from a limited set of initial data points. Left: Mean residual deviation error statistics for the GPR model in the analytic test system.

The total entropy of $M 1-\mathrm{VO}_{2}$ is $S_{\mathrm{M} 1}=S_{\mathrm{M} 1}^{\mathrm{ph}}$, and the total entropy for $R-\mathrm{VO}_{2}$ is $S_{\mathrm{R}}=S_{\mathrm{R}}^{\mathrm{el}}+S_{\mathrm{R}}^{\mathrm{ph}}+\tilde{S}_{\mathrm{R}}^{\mathrm{ph}} \cdot S_{\mathrm{R}}^{\mathrm{ph}}$ is the vibration contribution from the $3 n-2$ phonon modes that are harmonic at low temperature. $\tilde{S}_{\mathrm{R}}^{\mathrm{ph}}$ is from the two bands that soften at the transition and is based on the frequencies transformed to $T_{\mathrm{c}}$.

\section{APPENDIX D: GAUSSIAN PROCESS REGRESSION}

Supervised learning has been used to interpolate phonon frequencies using the nonparametric multivariate Bayesian method Gaussian process regression (GPR) [42]. GPR models can provide an appropriate alternative to Fourier interpolation, which is otherwise the method of choice when the full dynamical matrix is known. Without knowledge of the dynamical matrix at the transition, GPR models can be used to directly interpolate frequencies in $\mathbf{q}$ space from limited $\tilde{\omega}(\mathbf{q})$ data points.

In this work have used GPR for the $\mathbf{q}$-space interpolation of the two renormalized soft modes in $R-\mathrm{VO}_{2}$. The mode frequencies at $T_{\mathrm{c}}$ are determined from experiment at limited high-symmetry wave vectors. GPR can be used to predict how $\tilde{\omega}(\mathbf{q})$ varies across the full Brillouin zone, making possible thermodynamic calculations for the high-temperature phase from limited high-temperature data points. To show that the GPR approach can adequately predict the full $\tilde{\omega}(\mathbf{q})$ surface from limited data points, we benchmark the accuracy of GPR interpolation on an analytic model.

Consider a vanadium-oxygen analytic model with the following dispersion relation:

$$
\begin{aligned}
& \tilde{\omega}\left(q_{x}, q_{y}\right) \\
& =\sqrt{\left(\frac{1}{m_{\mathrm{O}}}+\frac{1}{m_{\mathrm{V}}}\right) \pm\left\{\left(\frac{1}{m_{\mathrm{O}}}+\frac{1}{m_{\mathrm{V}}}\right)^{2}-\frac{4}{m_{\mathrm{O}} m_{\mathrm{V}}} \sin ^{2} \mathbf{q}\right\}},
\end{aligned}
$$

which is shown in Fig. 5. The test system includes features such as optic and acoustic-type dispersion, with frequencies that are nonlinear in wave vector in more than one dimension and that have stationary points of inflection. The system is therefore expected to provide meaningful accuracy benchmarks, while also being simple enough to clearly illustrate the method.

To test if the GPR method can reproduce $\tilde{\omega}\left(q_{x}, q_{y}\right)$ from partial data, we make a training set consisting of 100 points/line along $q_{y} \in\left[0, \frac{\pi}{2}\right]$, at $q_{x}=0$ and $q_{x}=\frac{\pi}{2}$. Root mean square (rms) residual errors of the interpolated system compared to the true system are 5\% for the acoustic band and $8 \%$ for the optic, with percentages calculated with respect to the maximum frequency value of $\tilde{\omega}=0.54$ at $\mathbf{q}=0$. Typically we also know frequency gradients at zone boundaries. For a more realistic test model, derivatives at boundaries are included in the training set. This lowers rms residual errors across $\mathbf{q}$ to $2 \%$ and $3 \%$ for the acoustic and optic bands respectively.

In the GPR applied in this work, for the soft modes in $R-\mathrm{VO}_{2}$, an analogous interpolation is made for the two transition bands in $\left\{q_{x}, q_{y}, q_{z}\right\}$. Errors of $2 \%$ for the interpolated $R-\mathrm{VO}_{2}$ soft modes correspond to errors of approximately $1 \%$ or $0.01 k_{\mathrm{B}} / \mathrm{VO}_{2}$ in the transition entropy difference, which is satisfactory within the scope of this work and in context of other sources of error.

To interpolate the $R-\mathrm{VO}_{2}$ soft modes we have used a GPR with a nondeterministic radial basis function kernel of the form

$$
k\left(q, q^{\prime}\right)=\sigma_{f}^{2} \exp \frac{-\left(q-q^{\prime}\right)^{2}}{2 l^{2}}+\sigma_{n}^{2} \delta\left(q, q^{\prime}\right),
$$

with Bayesian maximum posterior $\theta=\left\{\sigma_{f}, \sigma_{n}, l\right\}$ hyperparameters. Training data includes line paths between highsymmetry points in the $q_{z}=\frac{1}{2}$ and $q_{z}=0$ planes, as well as zone-boundary band velocities. GPR training data for soft 
modes in the $q_{z}=\frac{1}{2}$ plane consists of renormalized harmonic frequencies. These are sampled at 100 points/line for each edge in the cycle $\{\mathbf{R}, \mathbf{Z}, \mathbf{A}, \mathbf{R}\}$. For the $q_{z}=0$ plane, in which the transition-mode bands do not soften at the transition, training data consist of 100 points/line samples of the edges in the $\{\boldsymbol{\Gamma}, \mathbf{X}, \mathbf{M}, \boldsymbol{\Gamma}\}$ graph for harmonic frequencies.
[1] T. D. Manning and I. P. Parkin, J. Mater. Chem. 14, 2554 (2004).

[2] M. Netsianda, P. E. Ngoepe, C. R. A. Catlow, and S. M. Woodley, Chem. Mater. 20, 1764 (2008).

[3] X. Tan, T. Yao, R. Long, Z. Sun, Y. Feng, H. Cheng, X. Yuan, W. Zhang, Q. Liu, C. Wu, Y. Xie, and S. Wei, Sci. Rep. 2, 466 (2012).

[4] C. Piccirillo, R. Binions, and I. P. Parkin, Thin Solid Films 516, 1992 (2008).

[5] P. Jin, S. Nakao, and S. Tanemura, Thin Solid Films 324, 151 (1998).

[6] M. E. A. Warwick and R. Binions, J. Mater. Chem. A 2, 3275 (2014).

[7] A. Tselev, I. A. Lukyanchuk, I. N. Ivanov, J. D. Budai, J. Z. Tischler, E. Strelcov, A. Kolmakov, and S. V. Kalinin, Nano Lett. 10, 4409 (2010).

[8] E. Merced, X. Tan, and N. Sepúlveda, Sens. Actuators, A 196, 30 (2013).

[9] J. R. Brews, Phys. Rev. B 1, 2557 (1970).

[10] F. Chudnovskiy, S. Luryi, and B. Spivak, Future Trends in Microelectriconics: The Nano Millenium (Wiley, New York, 2002), p. 148.

[11] B. T. O'Callahan, A. C. Jones, J. Hyung Park, D. H. Cobden, J. M. Atkin, and M. B. Raschke, Nat. Commun. 6, 6849 (2015).

[12] A. Cavalleri, C. Tóth, C. W. Siders, J. A. Squier, F. Ráksi, P. Forget, and J. C. Kieffer, Phys. Rev. Lett. 87, 237401 (2001).

[13] W. Klemm and L. Grimm, Naturwissenschaften (1913-2014) 27, 787 (1939).

[14] O. A. Cook, J. Am. Chem. Soc. 69, 331 (1947).

[15] F. J. Morin, Phys. Rev. Lett. 3, 34 (1959).

[16] A. Zylbersztejn and N. F. Mott, Phys. Rev. B 11, 4383 (1975).

[17] D. Paquet and P. Leroux-Hugon, Phys. Rev. B 22, 5284 (1980).

[18] R. Eguchi, M. Taguchi, M. Matsunami, K. Horiba, K. Yamamoto, Y. Ishida, A. Chainani, Y. Takata, M. Yabashi, D. Miwa, Y. Nishino, K. Tamasaku, T. Ishikawa, Y. Senba, H. Ohashi, Y. Muraoka, Z. Hiroi, and S. Shin, Phys. Rev. B 78, 075115 (2008).

[19] R. M. Wentzcovitch, W. W. Schulz, and P. B. Allen, Phys. Rev. Lett. 72, 3389 (1994).

[20] H. Zheng and L. K. Wagner, Phys. Rev. Lett. 114, 176401 (2015).

[21] S. Biermann, A. Poteryaev, A. Lichtenstein, and A. Georges, Phys. Rev. Lett. 94, 026404 (2005).

[22] S. Wall, S. Yang, L. Vidas, M. Chollet, J. M. Glownia, M. Kozina, T. Katayama, T. Henighan, M. Jiang, T. A. Miller, D. A. Reis, L. A. Boatner, O. Delaire, and M. Trigo, Science 576, 572 (2018).

[23] H. Wang, T. A. Mellan, R. Grau-Crespo, and U. Schwingenschlögl, Chem. Phys. Lett. 608, 126 (2014).

[24] B. Xiao, J. Sun, A. Ruzsinszky, and J. P. Perdew, Phys. Rev. B 90, 085134 (2014).

[25] J. P. Perdew, K. Burke, and M. Ernzerhof, Phys. Rev. Lett.77, 3865 (1996).
[26] S. L. Dudarev, G. A. Botton, S. Y. Savrasov, C. J. Humphreys, and A. P. Sutton, Phys. Rev. B 57, 1505 (1998).

[27] The on-site Coulomb interaction could be tuned arbitrarily to reproduce the experimental latent heat of transition of $44 \mathrm{meV} / \mathrm{VO}_{2}$, but such tuning is beyond the scope of this paper.

[28] R. Grau-Crespo, H. Wang, and U. Schwingenschlögl, Phys. Rev. B 86, 081101 (2012).

[29] V. Eyert, Phys. Rev. Lett 107, 016401 (2011).

[30] A. I. Duff, T. Davey, D. Korbmacher, A. Glensk, B. Grabowski, J. Neugebauer, and M. W. Finnis, Phys. Rev. B 91, 214311 (2015).

[31] B. Grabowski, L. Ismer, T. Hickel, and J. Neugebauer, Phys. Rev. B 79, 134106 (2009).

[32] O. Hellman, P. Steneteg, I. A. Abrikosov, and S. I. Simak, Phys. Rev. B 87, 104111 (2013).

[33] F. Zhou, W. Nielson, Y. Xia, and V. Ozolinš, Phys. Rev. Lett. 113, 185501 (2014).

[34] J. C. A. Prentice and R. J. Needs, Phys. Rev. Mater. 1, 023801 (2017).

[35] B. Monserrat, N. D. Drummond, and R. J. Needs, Phys. Rev. B 87, 144302 (2013).

[36] H. Terauchi and J. B. Cohen, Phys. Rev. B 17, 2494 (1978).

[37] W. Cochran, Phys. Rev. Lett. 3, 412 (1959).

[38] W. Cochran, Adv. Phys. 10, 401 (1961).

[39] W. Cochran, Ferroelectrics 35, 3 (1981).

[40] R. A. Cowley, Adv. Phys. 29, 1 (1980).

[41] W. Cochran F.R.S., The Dynamics of Atoms in Crystals, 1st ed., edited by B. Coles (Edward Arnolds Limited, London, 1973), p. 145.

[42] C. E. Rasmussen and C. K. I. Williams(MIT Press, Cambridge, MA, 2006).

[43] C. N. Berglund and H. J. Guggenheim, Phys. Rev. 185, 1022 (1969).

[44] J. D. Budai, J. Hong, M. E. Manley, E. D. Specht, C. W. Li, J. Z. Tischler, D. L. Abernathy, A. H. Said, B. M. Leu, L. A. Boatner, R. J. McQueeney, and O. Delaire, Nature (London) 515, 535 (2014).

[45] Y. Xia and M. K. Y. Chan, arXiv:1711.02819v3.

[46] T. Kawakubo, J. Phys. Soc. Jpn. 20, 516 (1965).

[47] W. Paul, Mater. Res. Bull. 5, 691 (1970).

[48] C. J. Hearn, J. Phys. C: Solid State Phys. 5, 1317 (1972).

[49] G. V. Chandrashekhar, H. L. C. Barros, and J. M. Honig, Mater. Res. Bull. 8, 369 (1973).

[50] F. Pintchovski, W. S. Glaunsinger, and A. Navrotsky, J. Phys. Chem. Solids 39, 941 (1978).

[51] D. Maurer, A. Leue, R. Heichele, and V. Müller, Phys. Rev. B 60, 13249 (1999).

[52] G. Kresse and J. Furthmüller, Phys. Rev. B 54, 11169 (1996).

[53] G. Kresse and J. Furthmüller, Comp. Mater. Sci. 6, 15 (1996).

[54] P. E. Blöchl, Phys. Rev. B 50, 17953 (1994).

[55] G. Kresse and D. Joubert, Phys. Rev. B 59, 1758 (1999).

[56] L. Chaput, A. Togo, I. Tanaka, and G. Hug, Phys. Rev. B 84, 094302 (2011). 